\title{
IMPLICATIONS OF SOLAR $p$-MODE FREQUENCY SHIFTS
}

\author{
Peter Goldreich, Norman Murray, and Gregory Willette \\ California Institute of Technology, Pasadena, CA 91125 \\ AND \\ PAWAN Kumar \\ High Altitude Observatory, National Center for Atmospheric Research, ${ }^{1}$ P.O. Box 3000, Boulder, CO 80307 \\ Received 1990 July 9; accepted 1990 September 18
}

\begin{abstract}
We relate entropy and magnetic field perturbations to variations of solar $p$-mode eigenfrequencies. The frequency variations result from changes in path length and propagation speed. These produce shifts of opposite sign. Path length changes dominate for entropy perturbations, and propagation speed changes dominate for most types of magnetic field perturbations. The $p$-mode frequencies increased along with solar activity between 1986 and 1989. The frequency shifts exhibit a rapid rise with increasing frequency followed by a precipitous drop. The positive component signals a strengthening of the photospheric magnetic field to an rms value of order $200 \mathrm{G}$. The sudden drop at high frequency is due to a combination of a resonance and an increase in temperature in the chromospheric cavity.

The magnetic stress perturbation decays above the top of the convection zone on a length scale comparable to the pressure scale height and grows gradually with depth below. The former characteristic implies that the stress is mainly due to small magnetic elements of the enhanced network, a conclusion supported by our analysis of Kitt Peak magnetograms. The latter property suggests that the flux tubes which pierce the photosphere strengthen with depth, at least to a pressure level of $10^{8}$ dynes $\mathrm{cm}^{-2}$.

The presence of a resonance in the chromospheric cavity means that the transition layer maintains enough coherence to partially reflect acoustic waves even near cycle maximum. The fractional chromospheric temperature rise implies a much larger fractional increase in the rate of mechanical heating, as indicated by the variation of the $\mathrm{Ca}$ II $\mathrm{H}$ and $\mathrm{K}$ lines.
\end{abstract}

Subject headings: hydromagnetics - Sun: oscillations

\section{INTRODUCTION}

Libbrecht \& Woodard (1990a, b) have discovered variations of the frequencies of the solar p-modes. Between 1986 and 1989, a period of increasing magnetic activity, the eigenfrequencies increased. The $m$-dependence of the frequency shifts convincingly relates them to perturbations concentrated in the latitudes of magnetic activity. Our goal is to identify the physical changes in the Sun that are responsible for the frequency shifts. The small rise in solar luminosity during 19861988 (Willson \& Hudson 1988) provides an important constraint. We proceed as follows.

In $\S 2$, we derive an expression that relates frequency shifts to changes in the entropy and magnetic field of the Sun. The changes that give rise to the measured frequency shifts are identified in $\S 3$. We consider the effects of magnetic activity on solar luminosity in $\S 4$. In the final section, $\S 5$, we compare our results to those obtained in recent papers by Campbell \& Roberts (1989) and Evans \& Roberts (1990). Then, we discuss some loose ends, including suggestions for future work, and summarize our main conclusions.

\section{DERIVATION OF FREQUENCY SHIFTS}

We concentrate on the simplest case, the adiabatic, radial modes of a nonrotating, spherical Sun. These simplifications deserve comment. Magnetic fields single out a particular direction at each point in space, and a magnetic field cannot be

\footnotetext{
${ }^{1}$ The National Center for Atmospheric Research is sponsored by the National Science Foundation.
}

spherically symmetric. Spherical symmetry can make sense as a statistical property of a small-scale magnetic field. However, this property does not characterize the Sun's magnetic activity which displays a strong latitude dependence that is reflected in the $m$-dependence of the frequency shifts.

We simplify because to do otherwise is not practical at this stage of investigation. There is an unwelcome degree of freedom in nonspherical solar models, especially those with magnetic fields. We describe the angle averaged magnetic field by two parameters, its mean square intensity, $B^{2}$, and an anisotropy parameter, defined later in this section and discussed in the Appendix. The restriction to radial modes, $l=0$, is not a serious one. The frequency shifts arise in the surface layers of the Sun, and the modes for which they are measured have sufficiently low $l$ that, in this region, their radial gradients are much larger than their angular gradients. Aside from a known correction due to the $l$-dependence of mode mass, we expect the frequency shifts calculated for radial modes to provide good estimates for the $m$-averaged frequency shifts of nonradial modes of similar frequency. This expectation is met by the $l$-dependence of the measured frequency shifts (Libbrecht \& Woodard 1990a, b).

A static solar model is specified by the functional dependence of its composition, entropy per unit mass, $s$, and mean square magnetic field, $B^{2}$, on the enclosed mass, $m$. Radial oscillations are described by giving the radii of the mass shells, $r(m, t)$, as functions of time, $t$.

Our objective is to relate frequency shifts, $\tilde{\Delta} \omega$, of the radial modes to changes in the solar interior specified by $\tilde{\Delta} s$ and 
$\widetilde{\Delta} B^{2} .^{2}$ We proceed by applying a variational formulation of magnetohydrodynamics (MHD) as outlined below. The use of variational principles in similar contexts is well documented, so the reader is referred to standard works where detailed derivations are provided.

Consider an inviscid, insulated, self-gravitating fluid of infinite electrical conductivity. The dependent variables that characterize the fluid are its velocity, $v$, density, $\rho$, pressure, $p$, gravitational potential, $\psi$, and magnetic field, $\boldsymbol{B}$. The equation of state is expressed in terms of the internal energy per unit mass, $e(\rho, s)$. Note that $p=\rho^{2}(\partial e / \partial \rho)_{s}$, and $c^{2}=(\partial p / \partial \rho)_{s}$.

The MHD equation of motion,

$$
\rho \frac{d v}{d t}=-\nabla p-\rho \nabla \psi+\frac{1}{4 \pi}(\nabla \times B) \times B,
$$

follows from application of Hamilton's principle to the Lagrangian

$$
L=\int d^{3} x \mathscr{L}=\int d^{3} x\left(\rho \frac{|\boldsymbol{v}|^{2}}{2}-\rho e-\rho \psi-\frac{|\boldsymbol{B}|^{2}}{8 \pi}-\frac{|\nabla \psi|^{2}}{8 \pi G}\right) .
$$

Poisson's equation,

$$
\nabla^{2} \psi=4 \pi G \rho,
$$

is obtained by varying $\psi$ independently of $\boldsymbol{x}$ in $\mathscr{L}$. Eulerian and Lagrangian implementations of the variation principle are described by Newcomb (1962). In both cases, the fundamental variation is to the position, $\boldsymbol{x}$, of each fluid element. The constraints implied by the conservation of mass, energy, and magnetic flux linking fluid circuits are included by relating the variations of $\rho, s$, and $\boldsymbol{B}$ to those of $\boldsymbol{x}$. These relations are local ones.

To reduce $\mathscr{L}$ to a form suitable for describing linear perturbations, we decompose the exact state, $\left(\rho^{*}, s^{*}, \boldsymbol{v}^{*}, \boldsymbol{B}^{*}, \psi^{*}\right)$, into the unperturbed state, $(\rho, s, \boldsymbol{v}, \boldsymbol{B}, \psi)$, and a perturbation obtained by displacing the position of each fluid element from $\boldsymbol{x}$ to $\boldsymbol{x}^{*}=\boldsymbol{x}+\boldsymbol{\xi}$. The linearized equations of motion for $\boldsymbol{\xi}$ follow from the part of $\mathscr{L}$ that is second-order in $\xi$. A systematic derivation of $\mathscr{L}_{2}$ is provided in Dewar (1970), to which we refer the reader for details. Since we are treating the normal modes of a conservative system, we assume a harmonic time dependence with radian frequency $\omega$, and take the spatial eigenfunctions $\xi$ and $\delta \psi$ to be real.

Radial, $l=0$, oscillations of a spherical Sun are governed by a simplified form of $\mathscr{L}_{2}$, namely,

$$
\begin{aligned}
\mathscr{L}_{2}= & \frac{\rho}{2} \omega^{2} \xi^{2}-\frac{\left(\rho c^{2}-p\right)}{2}\left[\frac{1}{r^{2}} \frac{\partial}{\partial r}\left(r^{2} \xi\right)\right]^{2}-\frac{p}{2}\left(\frac{\partial \xi}{\partial r}\right)^{2}-p\left(\frac{\xi}{r}\right)^{2} \\
& +\frac{B_{r}^{2}}{4 \pi}\left[r^{1 / 2} \xi \frac{\partial}{\partial r}\left(\frac{\xi}{r^{3 / 2}}\right)\right]-\frac{B_{h}^{2}}{8 \pi}\left(\frac{\partial \xi}{\partial r}\right)^{2} \\
& +\frac{\rho}{r}\left(\frac{\partial \psi}{\partial r}-2 \pi G \rho\right) \xi^{2}-\rho \xi \frac{\partial \delta \psi}{\partial r}-\frac{1}{8 \pi G}\left(\frac{\partial \delta \psi}{\partial r}\right)^{2}
\end{aligned}
$$

Here $\delta \psi$ is the Eulerian potential perturbation. The magnetic field enters $\mathscr{L}_{2}$ through the squares of its radial and horizontal component, $B_{r}^{2}$ and $B_{h}^{2}$. The term proportional to $B_{r}^{2}$ is small compared to that proportional to $B_{h}^{2}$ in the relevant limit $|\xi / r| \ll|\partial \xi / \partial r|$.

\footnotetext{
${ }^{2}$ The tilde, ; on $\Delta$ denotes a variation associated with a perturbation of the static solar model.
}

The quadratic Lagrangian given in equation (4) differs in a number of ways from those commonly applied in investigations of radial oscillations of stars (see Ledoux \& Walraven 1958). It accounts for the magnetic stress, and it treats the gravitational field as a dynamical variable. Dropping the magnetic terms and substituting $\partial \delta \psi / \partial r=-4 \pi G \rho \xi$ brings our expression to within a divergence of the standard form. Of course, the Euler-Lagrange equation obtained from this reduced form of $\mathscr{L}_{2}$ is precisely the standard wave equation for radial oscillations.

We drop the gravitational self-energy term from here on, ${ }^{3}$ since our interest is in high-order, $n \gg 1$, modes for which the gravitational perturbation is negligible. The Euler-Lagrange equation obtained from $\mathscr{L}_{2}$ has the Sturm-Liouville form. Multiplying this equation by $\xi$ and integrating over the volume of the Sun, we verify that $L_{2}=0$ for the normal modes. The equality of kinetic and potential energies, and the consequent vanishing of the action, is a general property of quadratic Lagrangians when evaluated on the solution curve.

We normalize the eigenfunctions according to

$$
4 \pi \omega \omega^{\prime} \int_{0}^{R} d r r^{2} \rho \xi_{\omega} \xi_{\omega^{\prime}}=\delta_{\omega, \omega^{\prime}},
$$

where the form of the weight function is dictated by the SturmLiouville equation. With this normalization,

$$
M_{\omega} \equiv \frac{1}{\omega^{2} \xi^{2}(R)}
$$

provides a measure of the mode mass in the sense that the energy, $E_{\omega}$, in the mode is related to the surface velocity amplitude, $v_{\omega}$, by

$$
E_{\omega}=M_{\omega} v_{\omega}^{2}
$$

We anticipate that significant contributions to $\tilde{\Delta} \omega$ are confined to the surface layers of the Sun. Entropy variations at depth are expected to be small since these layers have large thermal inertia. Also, estimates of the magnetic stress suggest that, deep in the convection zone, it is much smaller than the thermal pressure. Accordingly, we neglect $4 \pi G \rho$ with respect to $\omega^{2}$, and $\widetilde{\Delta} r / r$ with respect to $\widetilde{\Delta} c / c$ and $\partial \tilde{\Delta} r / \partial r$. Since the action is stationary under small variations of $\xi$ about an eigenfunction, equation (4) yields

$$
\frac{\tilde{\Delta} \omega}{\omega} \approx 2 \pi \int_{0}^{R} d r r^{2} \rho\left[\left(\tilde{\Delta} c^{2}+\tilde{\Delta} v_{A}^{2}\right)-2\left(c^{2}+v_{A}^{2}\right) \frac{\partial \tilde{\Delta} r}{\partial r}\right]\left(\frac{\partial \xi}{\partial r}\right)^{2}
$$

where

$$
v_{A}^{2}=\frac{B_{h}^{2}}{4 \pi \rho}
$$

is the square of the Alfvén speed. The term proportional to $\widetilde{\Delta} c^{2}+\widetilde{\Delta} v_{A}^{2}$ accounts for the change in propagation speed, and that proportional to $\partial \tilde{\Delta} r / \partial r$ to the change in path length.

All that remains is to relate $\widetilde{\Delta} c^{2} / c^{2}, \tilde{\Delta} v_{A}^{2} / v_{A}^{2}$, and $\partial \tilde{\Delta} r / \partial r \approx$ $-\tilde{\Delta} \rho / \rho$ to $\tilde{\Delta} B_{h}^{2}$. The starting point for this calculation is the equation of hydrostatic equilibrium,

$$
p+\beta \frac{B^{2}}{8 \pi}=\frac{G M(M-m)}{4 \pi R^{4}}
$$

\footnotetext{
${ }^{3}$ This approximation was introduced by Cowling (1941).
} 


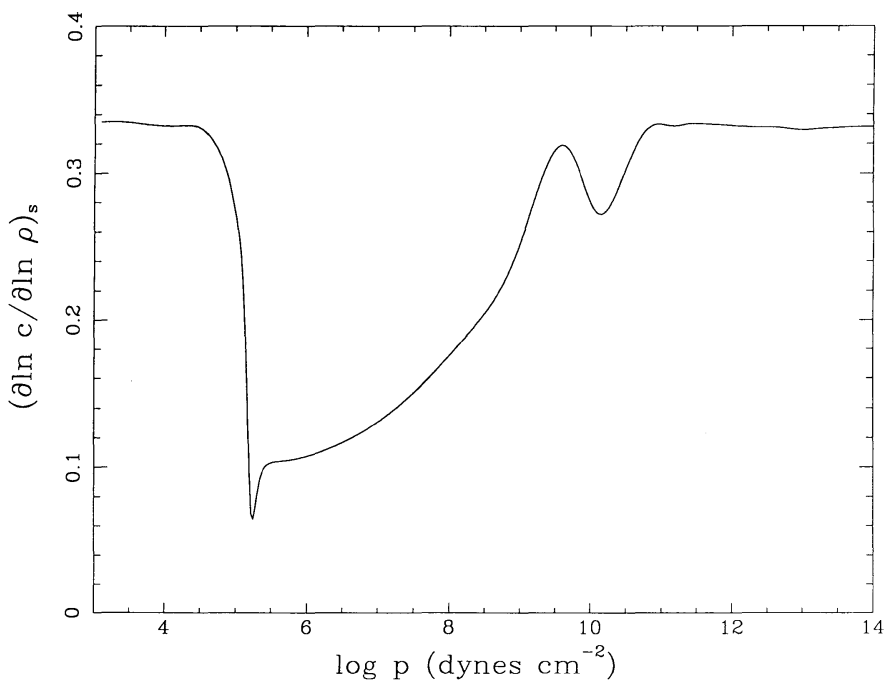

FIG. $1 a$

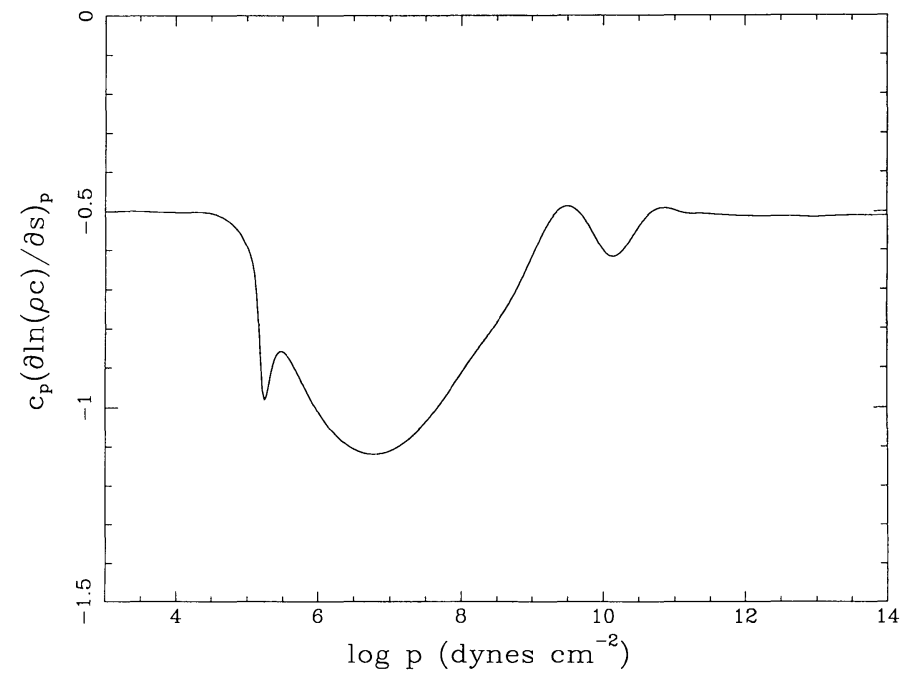

FIG. $1 b$

FIG. 1.-(a) The double logarithmic derivative of sound speed with respect to density taken at fixed specific entropy, plotted as a function of the logarithm of the

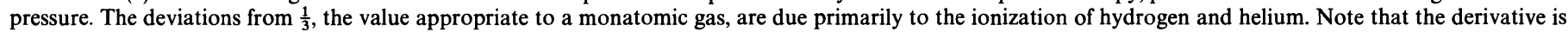

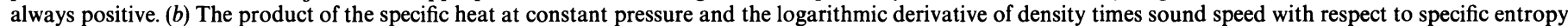

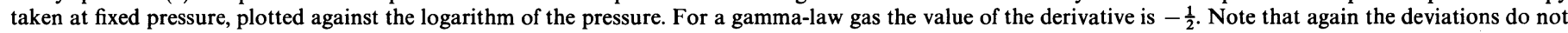
change the sign of the derivative.

written in a form suitable for a plane-parallel layer. ${ }^{4}$ Here $M$ is the solar mass and $G M / R^{2}$ is the surface gravitational acceleration. The coefficient $\beta$ accounts for the statistical anisotropy of the magnetic field (see Appendix); $\beta=-1$ for a purely radial field, $\beta=0$ for a force-free field, $\beta=\frac{1}{3}$ for a statistically isotropic small scale field, and $\beta=1$ for a purely horizontal field. We assume that $\beta$ is a constant independent of both position and time. Considering variations at fixed $m$, and using the equation of state, yields

$$
\begin{gathered}
\frac{\tilde{\Delta} \rho}{\rho}=\left(\frac{\partial \ln \rho}{\partial s}\right)_{p} \tilde{\Delta} s-\beta \frac{\tilde{\Delta} B^{2}}{8 \pi \rho c^{2}} \\
\widetilde{\Delta c^{2}}=\left(\frac{\partial c^{2}}{\partial s}\right)_{p} \tilde{\Delta s}-\left(\frac{\partial \ln c^{2}}{\partial \ln \rho}\right)_{s} \beta \frac{\widetilde{\Delta} B^{2}}{8 \pi \rho},
\end{gathered}
$$

and

$$
\widetilde{\Delta} v_{A}^{2}=-v_{A}^{2} \frac{\bar{\Delta} \rho}{\rho}+(1+\beta) \frac{\tilde{\Delta} B^{2}}{8 \pi \rho} .
$$

All the ingredients needed to relate $\bar{\Delta} \omega$ to $\bar{\Delta} s$ and $\widetilde{\Delta} B^{2}$ are now in hand. A straightforward calculation, discarding terms proportional to $v_{A}^{2} / c^{2} \ll 1$, yields

$$
\frac{\tilde{\Delta} \omega}{\omega} \approx 4 \pi \int_{0}^{R} d r r^{2} \rho c^{2} W(r),
$$

where the dimensionless weight factor

$$
\begin{aligned}
W(r)=\left\{\left[\frac{\partial \ln (\rho c)}{\partial s}\right]_{p}\right. & \tilde{\Delta}_{s} \\
+ & {\left.\left[\frac{1-\beta}{2}-\beta\left(\frac{\partial \ln c}{\partial \ln \rho}\right)_{s}\right] \frac{\tilde{\Delta} B^{2}}{8 \pi \rho c^{2}}\right\}\left(\frac{\partial \xi}{\partial r}\right)^{2} . }
\end{aligned}
$$

Equations (14) and (15) express our principal theoretical result. The effects of perturbations of path length and propagation

\footnotetext{
${ }^{4}$ We take $B=0$ at $p=0$.
}

speed are intertwined in $W(r)$. However, they are easily separated by use of equations (11), (12), and (13). Without path length changes, the $\rho$ would be missing in the coefficient of $\widetilde{\Delta} s$ and $1-\beta$ would be changed to $1+\beta$ in the coefficient of $\widetilde{\Delta} B^{2}$.

Figure 1 displays $c_{p}(\partial \ln (\rho c) / \partial s)_{p}$ and $(\partial \ln c / \partial \ln \rho)_{s}$ plotted against $\log p$ for the solar model of Christensen-Dalsgaard (1982). For a $\Gamma$-law equation of state, $c_{p}(\partial \ln (\rho c) / \partial s)_{p}=-\frac{1}{2}$, where $c_{p}$ is the specific heat per unit mass at constant pressure, and $(\partial \ln c / \partial \ln \rho)_{s}=(\Gamma-1) / 2$. Because of the presence of ionization zones, a $\Gamma$ law is a poor approximation to the equation of state in the upper part of the convection zone. However, the ionization zones do not affect the signs of $c_{p}(\partial \ln (\rho c) / \partial s)_{p}$ and $(\partial \ln c / \partial \ln \rho)_{s}$.

Entropy variations induce variations of like sign in both propagation speed and path length. However, the path length has the more important effect on the frequency shift. Consequently, entropy variations force frequency changes of opposite sign.

For magnetic field variations the situation is ambiguous. Changes of magnetic field strength produce changes of like sign in propagation speed. These are entirely due to the horizontal component of the field. The normal vertical magnetic stress is the net of the pressure from the horizontal component and the tension from the vertical component. It may be positive or negative. Thus, path length changes may be in the same or opposite sense from those of the magnetic field strength. However, from here on we assume that perturbations in magnetic field strength produce frequency shifts of similar sign, since this is true for $\beta<1 /\left[1+2(\partial \ln c / \partial \ln \rho)_{s}\right]$, that is, unless the field is nearly horizontal.

\section{PERTURBATIONS DUE TO MAGNETIC ACTIVITY}

Our numerical calculations are based on the solar model of Christensen-Dalsgaard (1982) whose outer boundary is at the temperature minimum. We extend this model by adding a chromosphere and corona following Vernazza, Avrett, \& Loeser (1981); we use their model C. This procedure involves some arbitrary smoothing where we join the two models. 
We calculate adiabatic eigenfunctions subject to the outer boundary condition that the Lagrangian pressure perturbation vanish at the base of the corona. The limitations of the adiabatic approximation are described in $\S 5$. The boundary condition provides an upper reflecting layer for modes of arbitrary frequency, thus ensuring they are trapped. It is strictly applicable in the limit that the corona has infinite temperature and vanishing density, but is quite adequate for all modes of interest to us. Calculations with an outward wave boundary condition in the corona give almost identical modes, except for the addition of small imaginary parts to the eigenfrequencies.

Our solar model has a chromospheric cavity as a consequence of our choice of outer boundary condition. Whether the solar transition layer is sufficiently homogeneous to act as a reflecting layer is an open issue. Magnetic stresses may exceed the gas pressure in some of this region during part of the time, so a considerable level of inhomogeneity seems likely. High-frequency modes present special calculational problems since, after tunneling through the temperature minimum, they begin to propagate again fairly low in the chromosphere. Thus, they are particularly sensitive to the structure of the chromosphere and to the position of the transition layer.

A technical comment is in order here. The envelopes of the $p$-mode eigenfunctions grow with increasing $r$. Thus, care must be exercised in deciding where it is safe to cut off the integral in equation (14). With our choice of outer boundary condition, it suffices to force the perturbations represented by $W$ to vanish at the outer boundary. Since the largest perturbations are expected to be in the outermost layers, this is not entirely realistic. In particular, the transition layer probably moves to higher pressure with increasing magnetic activity. To include this change, it is necessary to retain a surface term in the variational principle.

Each radial $p$-mode propagates in an inner cavity bounded by $r=0$ and a reflecting layer located somewhere below the temperature minimum. In this cavity, the envelope of its displacement eigenfunction, $\hat{\xi}$, satisfies the WKBJ relations

$$
r^{2} \rho c \xi^{2} \sim K,
$$

where $K$ is a constant, and

$$
\frac{\partial \hat{\xi}}{\partial r} \sim \frac{\omega}{c} \hat{\xi}
$$

The mode is evanescent in a region centered on the temperature minimum, and here its amplitude increases exponentially with height. The scale

$$
\alpha_{\omega} \sim \frac{\gamma g}{\omega^{2}}
$$

characterizes both the depth below the temperature minimum of the reflecting layer and the $e$-folding length in the evanescent region. Here, $\gamma$ is the effective adiabatic index for acoustic waves. $^{5}$

\subsection{Locations of Perturbations}

Fractional frequency shifts, $\tilde{\Delta} \omega / \omega$, between 1986 and 1989 are plotted in Figure 2 as a function of $\omega$. Two features are worth noting, the rapid rise of $\tilde{\Delta} \omega / \omega$ at low $\omega$ and its precipitous decline at high $\omega$. This pattern points to the locations of the sources of the frequency shifts. In future discussion, we

\footnotetext{
${ }^{5}$ The $\omega$ dependence of $\alpha$ is more complex very close to the acoustic cutoff.
}

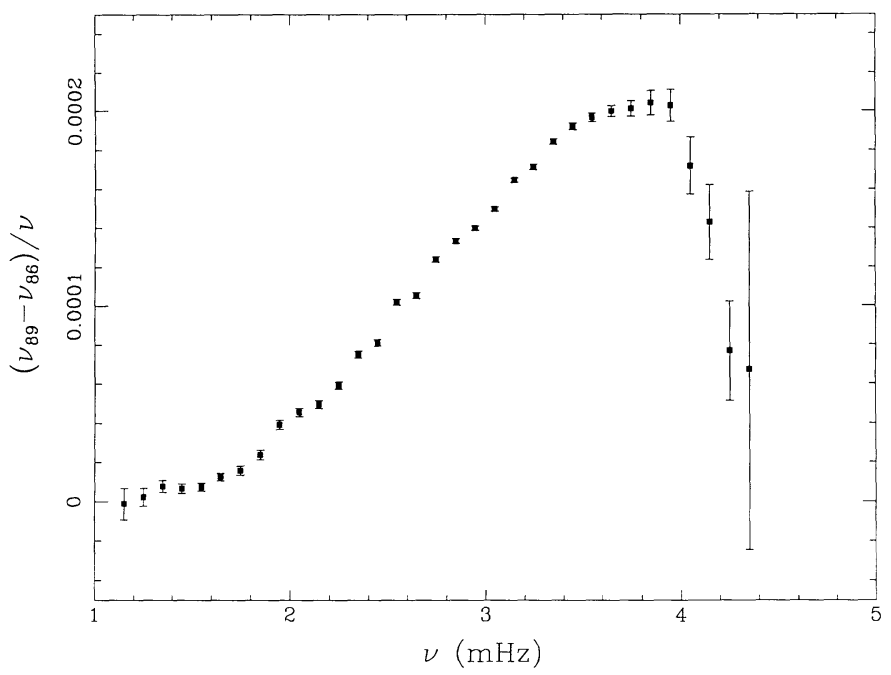

FIG. 2.-The fractional frequency shifts between 1989 and 1986, as measured by Libbrecht \& Woodard (1990a, b), plotted against frequency in $\mathrm{mHz}$. The frequency differences are averages over all measured modes in a frequency range of $0.1 \mathrm{mHz}$

assume that the frequency shifts can be decomposed into two components, a positive one at low frequency and a negative one at high frequency. Of course, this decomposition is not unique.

From the normalization equation (5) and the WKBJ relations (16) and (17), it follows that $\partial \xi / \partial r$ is independent of $\omega$ where the modes propagate. Then, equation (14) implies that $\widetilde{\Delta} \omega / \omega$ due to perturbations in a region where all modes propagate would be independent of $\omega^{6}$ Since $\tilde{\Delta} \omega / \omega \propto \omega^{6}$ at low frequencies, the perturbations are located above the upper reflecting layers of the lowest frequency modes for which shifts have been detected.

Photospheric perturbations would produce $\tilde{\Delta} \omega / \omega \propto$ $\omega^{2} / \boldsymbol{M}_{\omega}$, as can be seen from equations (6) and (14). This gives too strong a frequency dependence at low frequency, but not by very much. Contributions from perturbations just below the photosphere would weaken this dependence; the fractional difference of $\xi$ for low- and high-frequency modes decreases with depth. We conclude that the positive contribution to the frequency shifts, which dominates at low frequency arises in a region close to and possibly including the photosphere.

The precipitous decline of $\widetilde{\Delta} \omega / \omega$ at high frequency must be due to perturbations located at heights well above the photosphere. Comparison of the high-frequency behavior of $\bar{\Delta} \omega / \omega$ seen in Figure 2 with that of $(\partial \xi / \partial r)^{2}$, drawn in Figure 3 for several pressure levels, indicates that the negative contributions to the frequency shifts come from perturbations located at pressure levels at least three orders of magnitude below the pressure at the photosphere.

\subsection{Magnitudes of Perturbations}

The perturbations responsible for the positive component of the frequency shifts are located where the $p$-modes are evanescent. In these regions, a crude but illustrative approximation is set

$$
\xi(r) \sim \xi(R) \exp \left[\frac{(r-R)}{\alpha_{\omega}}\right],
$$

\footnotetext{
6 This statement assumes that the perturbations are sufficiently smooth that we may replace $\partial \xi / \partial r$ by its envelope in eq. (14).
} 


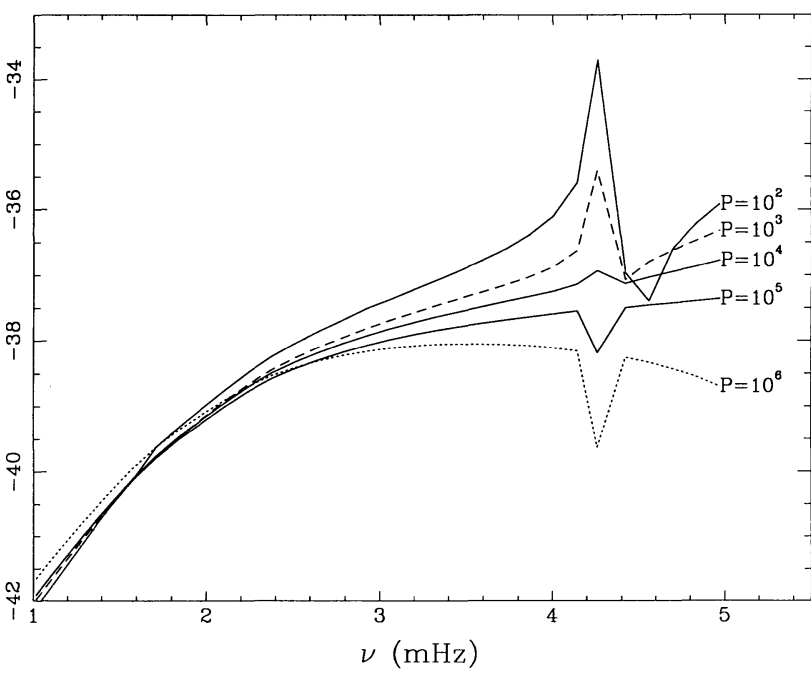

Fig. 3.-The logarithm of the square of the derivative of the radial displacement, plotted as a function of the frequency, for several different pressures in dynes $\mathrm{cm}^{-2}$. The solid lines are for pressures of $10^{2}, 10^{4}$, and $10^{5}$, as labeled. The dashed line is for a pressure of $10^{3}$, and the dotted line for a pressure of $10^{6}$. The spikes at $4.2 \mathrm{mHz}$ are due to a resonance in the chromospheric cavity.

where $\alpha_{\omega}$ is given by equation (18). The surface (photospheric) displacement, $\xi(R)$, is related to the mode mass by equation (6).

Next, we apply equations (14) and (19) to obtain the fractional frequency shift associated with a constant value of $W(r)$ maintained over a single pressure scale height, $H(r)$, centered at $r$. A straightforward calculation yields

$$
\frac{\Delta \omega}{\omega} \sim \frac{4 \pi \omega^{2} \rho R^{2} H^{2}}{\gamma g M_{\omega}} W \exp \left[\frac{2 \omega^{2}}{\gamma g}(r-R)\right],
$$

where $\rho, H$, and $W$ depend on $r$.

To obtain a feeling for the order of magnitude required of the perturbations, we evaluate $\Delta \omega / \omega$ for photospheric perturbations, and frequencies close to the peak of the $p$-mode excitation spectrum, $\omega \approx 2 \times 10^{-2} \mathrm{~s}^{-1}$. Taking $M_{\omega} \approx 1.5 \times 10^{25} \mathrm{~g}$, we find $\delta \omega / \omega \approx 10^{-3} W(R)$. Since the fractional frequency shifts near the 5 minute peak exceed $10^{-4}$, the dimensionless perturbation strength, as measured by $W$, must be of order $10^{-1}$ if the perturbations are confined to a single scale height. In fact, we show below that good fits to the data are obtained for dimensionless perturbations of order $10^{-2}$ that extend over several scale heights.

The effect on $\tilde{\Delta} \omega / \omega$ of entropy perturbations of constant magnitude declines exponentially with height since $H<\alpha_{\omega} / 2$ for trapped modes. However, the contribution to $\delta \omega / \omega$ by magnetic field perturbations of constant strength grows exponentially with height. The latter statement seems to imply that arbitrarily weak fields could have substantial effects if they extend far enough above the photosphere. However, the validity of equations (14) and (20) demands that the fields represent small perturbations to the solar model. This is equivalent to the restriction that $|W| \ll 1$.

As stated above, the limit on magnetically induced frequency shifts is imposed by the requirement that perturbation theory be a valid approximation. However, our experience with nonperturbative calculations suggests that it is more fundamental. For example, it is easy to assess frequency changes caused by a force free field of arbitrary strength, since force-free fields do not affect the static structure of the Sun. We find that these fields saturate the radial growth of mode eigenfunctions where
$v_{A} \gtrsim c$, and that this limits their total effect on the mode frequencies to the level indicated above. It is our unconfirmed opinion that such saturation limits the frequency shifts caused by sunspots to an insignificant level.

\subsection{Nature of Perturbations}

We know that the source of the positive-frequency shifts is located in the vicinity of the photosphere. However, we have yet to determine whether decreases in entropy or increases in magnetic field strength are the important perturbations.

The argument against pure entropy perturbations is a simple one. Although there is no difficulty in finding perturbations that reproduce the observed positive shifts, they all imply that between 1986 and 1989 the temperature just below the photosphere dropped by of order $1 \%$. Try as we might, we have been unable to reconcile this large temperature drop with the much smaller, $\lesssim 0.025 \%$, increase of the Sun's effective temperature derived by extrapolating the ACRIM data set (Wilson \& Hudson 1988). ${ }^{7}$ We return to this point in $\S 4$.

It is also easy to fit the observed positive frequency shifts with pure magnetic perturbations. Figure 4 shows a run of the rms magnetic field strength increase that fits the shifts between 1986 and 1989. It is computed on the basis that the magnetic field is random, that is, $\beta=\frac{1}{3}$. If instead, the field is force-free, its strength is reduced.

Published estimates of the variation of the Sun's rms magnetic field strength with solar cycle are hard to come by. Fortunately, this quantity may be derived from whole disk magnetograms, albeit in a somewhat model-dependent fashion. The procedure is to assume a standard value, $B_{m}$, for the strength of small-scale magnetic field elements; observations suggest that $B_{m} \approx 2 \times 10^{3} \mathrm{G}$ (Stenflo 1989). Then the mean absolute radial field strength, $\left|B_{r}\right|$, is measured from the the central portion of whole disk magnetograms. Because of

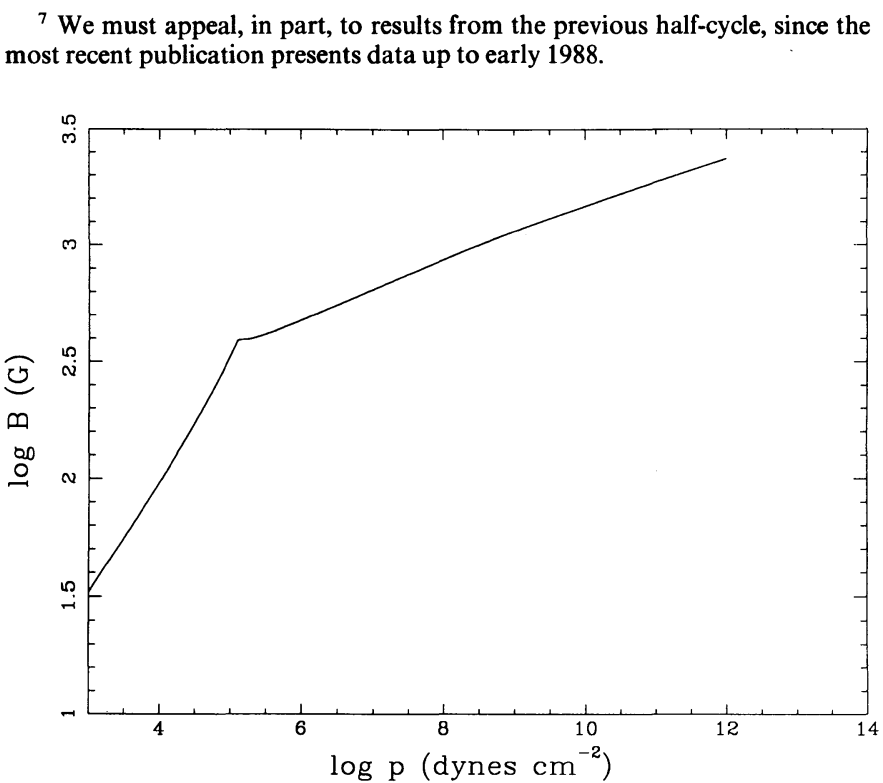

FIG. 4.-The root mean square magnetic field deduced from the observed frequency shifts. The logarithm of the rms field is plotted against the logarithm of the pressure. The square of the field increases with the one-third power of the density below the top of the convection zone, at $p=1.3 \times 10^{5}$ dynes cm $^{-2}$, and decays exponentially with a scale height of $220 \mathrm{~km}$ above the top of the convection zone. At the photosphere, $p \approx 9.0 \times 10^{4}$ dynes $\mathrm{cm}^{-2}$, the rms field is about $250 \mathrm{G}$. 
cancellation of unresolved fields of opposite polarity, this step yields a lower bound to $\left\langle\left|B_{r}\right|\right\rangle$. Ignoring this fine point, we have

$$
\left\langle B_{r}^{2}\right\rangle=\left\langle\left|B_{r}\right|\right\rangle B_{m} .
$$

We have applied this method to two Kitt Peak magnetograms, one from 1986 and the other from 1988. The results are $\left\langle B_{r}^{2}\right\rangle\left\langle(120 \mathrm{G})^{2}\right.$ in 1986 , and $\left\langle B_{r}^{2}\right\rangle \approx(190 \mathrm{G})^{2}$ in $1988^{8}$ The value of of $\left\langle B_{r}^{2}\right\rangle$ from 1988 was dominated by small-scale fields with sunspots making a relatively minor contribution. The difference in $\left\langle B_{r}^{2}\right\rangle$ between 1988 and 1986 is compatible with the value of $\widetilde{\Delta} B^{2}$ deduced from the frequency shifts during this time interval.

Our estimates of the rms value of the Sun's magnetic field implies that magnetic flux tubes cover about $1 \%$ of the Sun's surface area near solar maximum. The gas pressure in these tubes is substantially lower than that in the surrounding field free regions. It would require an unexpected coincidence for the specific entropy to be the same within and outside the tubes. Thus, an entropy perturbation must accompany the magnetic perturbation that we hold accountable for the positive component of the frequency shifts. ${ }^{9}$ This entropy perturbation contributes an unknown fraction of the frequency shifts.

The decline of $\bar{\Delta} \omega$ above $v \approx 4 \mathrm{mHz}$ must be due to an entropy increase in the optically thin layers of the solar atmosphere. The alternative explanation, a decrease of the magnetic field strength during a time of increasing magnetic activity, is untenable. The precipitous nature of the decline results from a chromospheric resonance which, for our solar model, occurs at $v \approx 4.4 \mathrm{mHz}$. Near resonant modes have large amplitudes in the chromosphere, so they are sensitive to changes in the temperature there. A typical example of a fractional temperature perturbation that can account for the observed behavior is shown in Figure 5. This example shares with all others the characteristic that its contributions to the frequency shifts are concentrated at pressure levels more than four orders of magnitude below that at the photosphere.

Both observational inaccuracy and calculational uncertainty limit our ability to determine the entropy perturbations responsible for the sudden drop in $\widetilde{\Delta} \omega / \omega$ at high $\omega$. The measurement of frequency shifts for high-frequency $p$-modes is made less accurate by the large line widths of these modes. Also, as discussed earlier, high-frequency modes are sensitive to the poorly determined and inhomogeneous structure of the outer chromosphere and transition layer. The parameters of the chromospheric resonance, its frequency and strength, are correlated with the temperature increase deduced from the negative component of the frequency shifts. It is likely that the transition layer is both nonspherical and time-dependent. A good fit to the data can be obtained with $\tilde{\Delta T / T}=0.01$ by fine-tuning the position of the transition layer to make the resonance frequency fall at $v=4.2 \mathrm{mHz}$.

Figure 6 shows the depth dependence of the contributions to the fractional frequency shifts of four modes by the perturbations displayed in Figures 4 and 5. The overall fit of the calculated shifts to the measured values is illustrated in Figure 7. Perusal of Figure 7 reveals two interesting features. The data points oscillate about the rising portion of the curve in a sys-

\footnotetext{
${ }^{8}$ The mean square radial field from 1986 is consistent with the noise level of the magnetogram.

${ }^{9}$ The entropy perturbation would be small if the tubes contain very little gas.
}

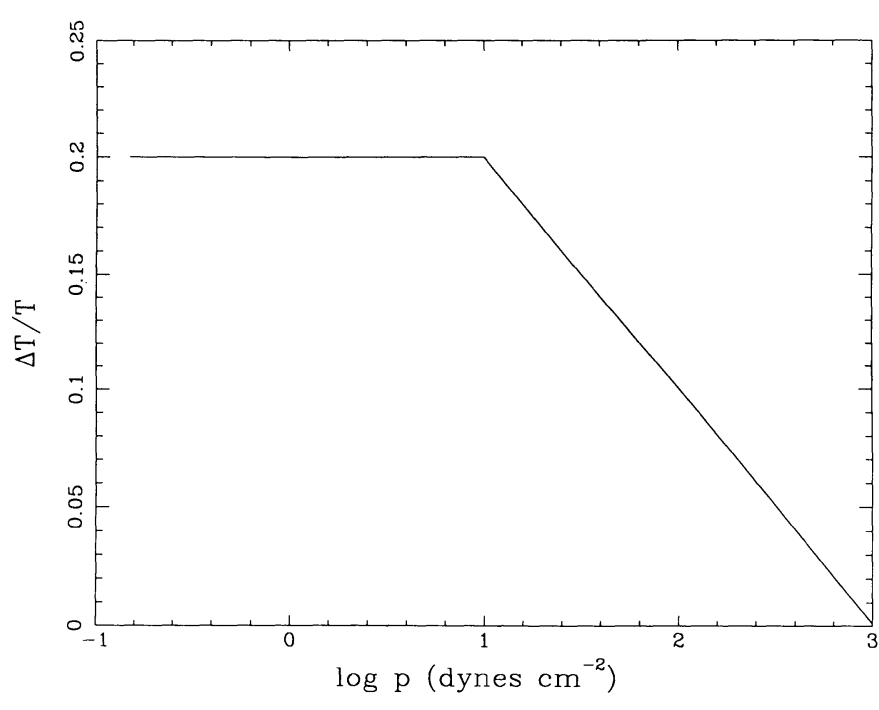

FIG. 5.-The fractional increase in chromospheric temperature from 1986 to 1989 , plotted against the logarithm of pressure. The temperature change reproduces the sharp cutoff in the frequency shifts shown in Fig. 2. Only its magnitude and location are significant; its shape is not.

tematic fashion. This hints that there is an unmodeled perturbation localized in radius. The oscillation of the residuals is a response to the shifting of the position of the nodes of $\partial \xi / \partial r$ with respect to that of the perturbation as $\omega$ varies (see $\S 5.1$ ). Also, following its precipitous drop, the curve fit to $\widetilde{\Delta} \omega / \omega$ exhibits a sharp rise as expected of a resonance. The bottom of the dip occurs at a frequency for which the eigenmode has a quarter-wavelength in the outer cavity.

\section{LUMINOSITY VARIATIONS}

In rejecting pure entropy perturbations as a cause of the positive component of the frequency shifts, we appealed to the small size of the solar cycle luminosity variations. This left magnetic perturbations as the only viable alternative. This choice was supported by our estimates from two Kitt Peak magnetograms of the mean square magnetic fields during 1986 and 1988. However, it remains a problem to reconcile the large increase we deduce for the magnetic energy density with the small observed luminosity increase.

The frequency shifts imply changes in the mean magnetic stress at and just below the photosphere that are of order $1 \%$ of the gas pressure. The eddy stress is believed to be about $10 \%$ of the gas pressure at the top of the convection zone. Thus, at cycle maximum the mean magnetic stress is of order $10 \%$ of the eddy stress. According to conventional wisdom, magnetic fields impede convection, that is, a larger superadiabatic temperature gradient is needed to transport a fixed energy flux in the presence of a magnetic field than in its absence. It is puzzling that the large increase of magnetic stress between cycle minimum and maximum is accompanied by a slight increase, rather than a more sizable decrease, of luminosity. A quantitative assessment follows.

Any theory that attempts to connect variations of solar luminosity with magnetic activity is speculative, since it must address the interaction of magnetic fields with convection. We base our discussion on the mixing length model of convection. Although independently conceived, our treatment is similar to that of Spruit (1989) and, in its final form, owes much to his work. 


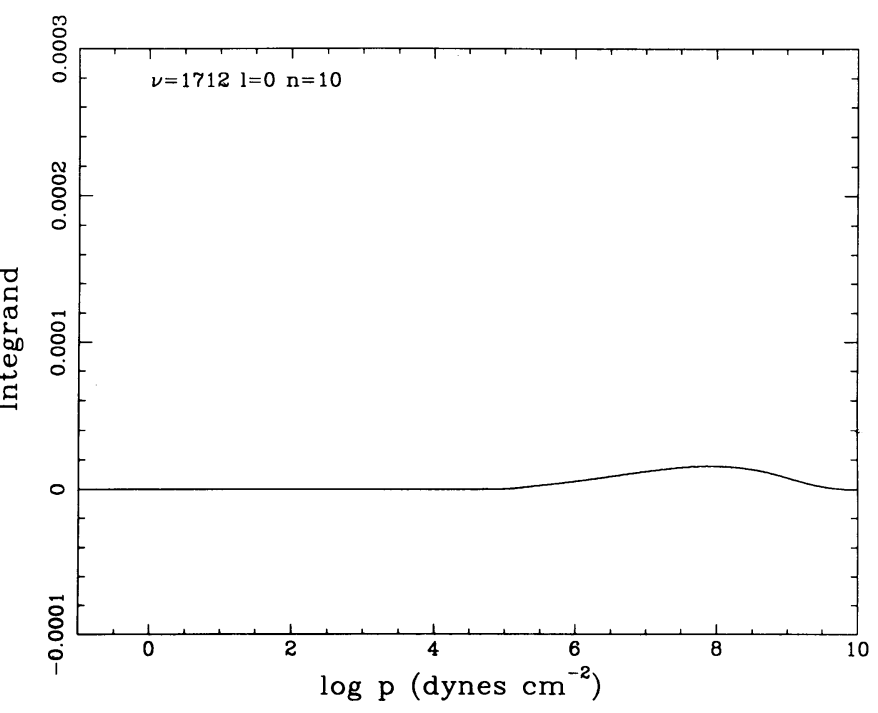

FIG. $6 a$

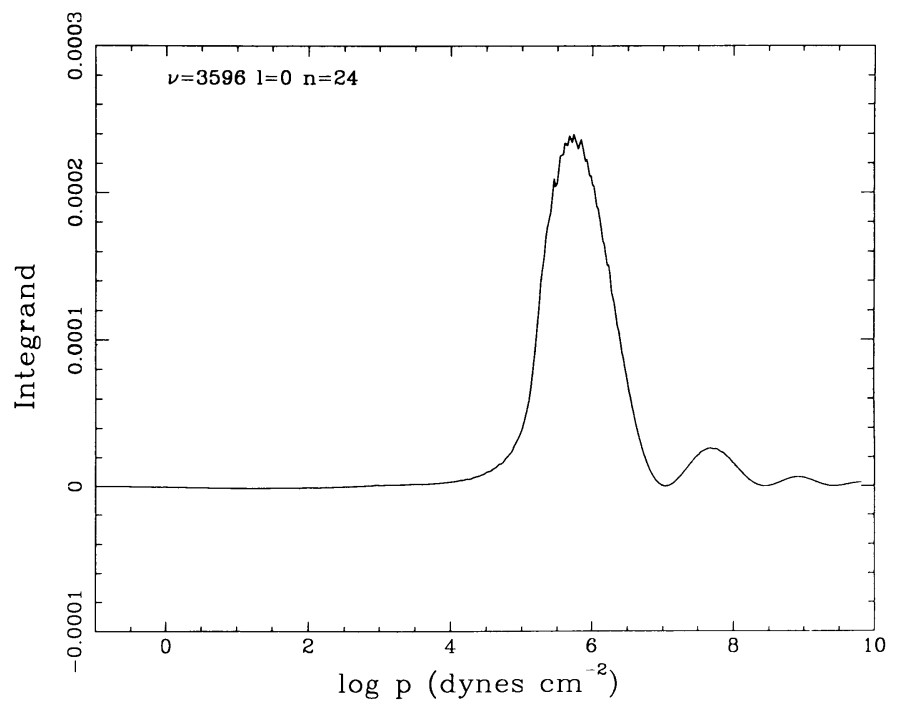

FIG. $6 c$

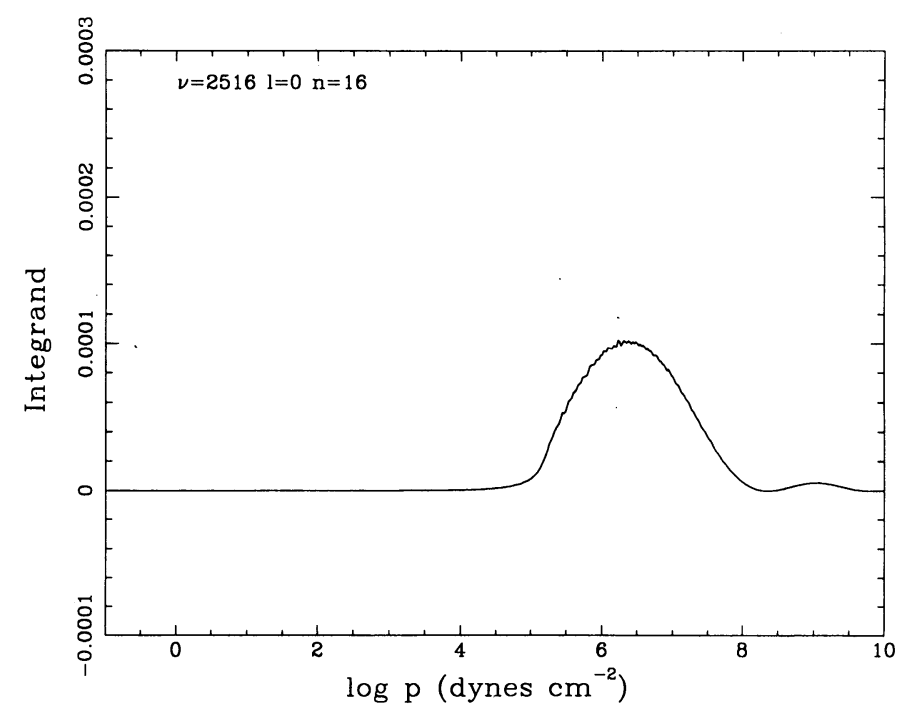

Fig. $6 b$

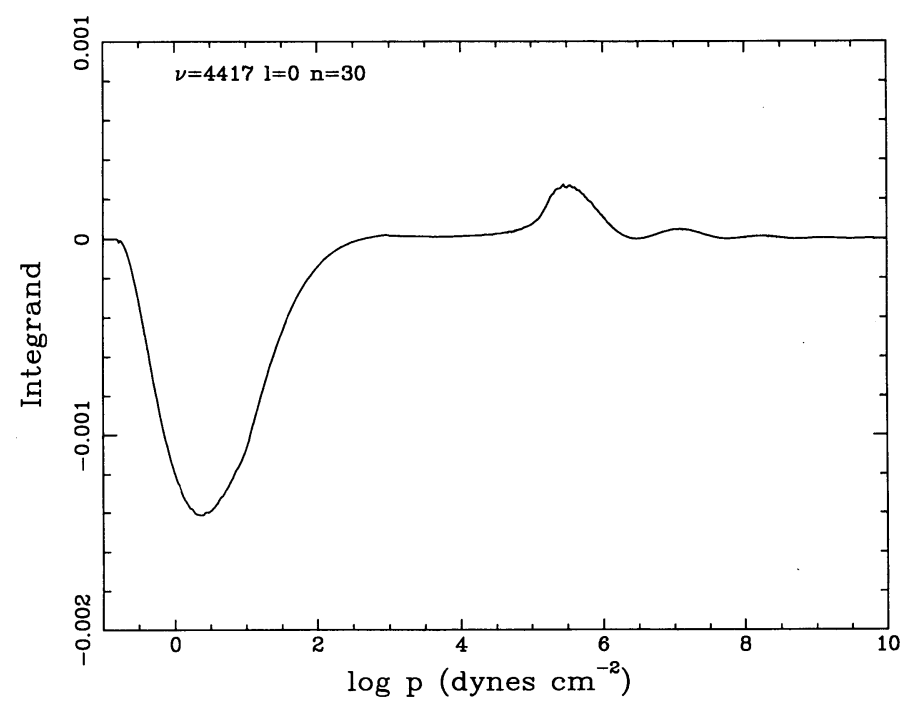

Fig. $6 d$

FIG. 6. - The integrand in the expression for the fractional frequency shift given by equation (14), plotted against the logarithm of the pressure, for four different modes designated by frequency in $\mathrm{mHz}, v$, and number of radial nodes, $n$, all with $l=0:(a) v=1712, n=10 ;(b) v=2516, n=16 ;(c) v=3596, n=24 ;(d) v=4417$, $n=30$. Note the different scale in $(d)$. The integrands use the magnetic field from Fig. 4 and the temperature shift from Fig. 5. The jagged appearance of the curves is due to the finite resolution of our solar model.

The mixing length model relates the convective energy flux, $F$, to the convective velocity, $v$, by

$$
F \sim \rho v^{3}
$$

and the entropy gradient, $\partial s / \partial r$, to the convective velocity and pressure scale height, $H$, by

$$
\frac{H}{c_{p}} \frac{\partial s}{\partial r} \sim-\left(\frac{v}{c}\right)^{2} .
$$

Combining these equations yields

$$
F \sim \rho c^{3}\left(-\frac{H}{c_{p}} \frac{\partial s}{\partial r}\right)^{3 / 2} .
$$

It is convenient to define the entropy deficit,

$$
\mathscr{D} S(r)=\int_{r_{b}}^{r} d r \frac{\partial s}{\partial r},
$$

as the difference between the specific entropy at $r$ and at the bottom of the convection zone, $r_{b}$. Note that $\mathscr{D} s(r)$ is a monotonically decreasing negative function of $r$ in the convection zone. Also, since the magnitude of $\partial s / \partial r$ increases rapidly with $r$

$$
H \frac{\partial s}{\partial r} \sim \mathscr{D} s
$$

The response to solar cycle modulation at different depths in the convection zone is characterized by two disparate time scales, the shorter diffusion time,

$$
\tau_{d} \sim \frac{H}{v},
$$

and the longer thermal relaxation time,

$$
\tau_{t} \sim \frac{H p}{F} .
$$




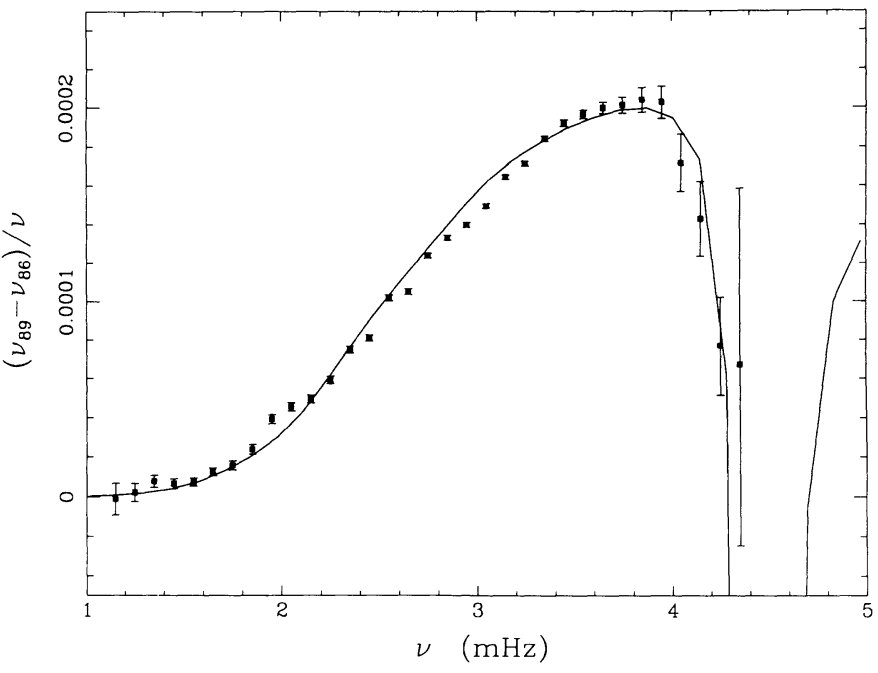

FIG. 7.-Fractional frequency shifts computed from equation (14), using the magnetic field from Fig. 4 and the temperature change from Fig. 5, compared to the measured values presented in Fig. 2.

These time scales are displayed as functions of pressure in Figure 8. Comparison with Figure 5 shows that the perturbations responsible for the positive component of the frequency shifts occur where both $\tau_{d}$ and $\tau_{t}$ are much shorter than the cycle period, $\tau_{c}$.

Since magnetic fields impede convection, we modify equation (23) so that it reads

$$
\frac{H}{c_{p}} \frac{\partial s}{\partial r} \sim-(1+\epsilon)\left(\frac{v}{c}\right)^{2},
$$

where

$$
\epsilon \sim \frac{B^{2}}{8 \pi \rho v^{2}}
$$

is the ratio of the mean magnetic stress to the eddy stress. Then, using equation (22), the perturbed entropy gradient is written as

$$
\frac{\partial \tilde{\Delta} s}{\partial r} \approx \frac{2}{3}\left(\frac{\tilde{\Delta} F}{F}+\tilde{\Delta} \epsilon\right) \frac{\partial s}{\partial r} .
$$

The perturbed energy equation,

$$
\rho T \frac{\partial \tilde{\Delta} s}{\partial t}=\frac{1}{r^{2}} \frac{\partial}{\partial r}\left(r^{2} \tilde{\Delta} F\right),
$$

relates $\tilde{\Delta} s$ to $\tilde{\Delta} F$. In writing equations (31) and (32), we neglect $\tilde{\Delta} \rho / \rho, \tilde{\Delta} c / c$, and $\tilde{\Delta} T / T$ with respect to $\tilde{\Delta} s / \mathscr{D} s$. The former are smaller than the latter by of order $\left|\mathscr{D} s / c_{p}\right|$. This quantity, which is a measure of convective efficiency, rises from small values at depth to approximately unity at the top of the convection zone, $r=r_{t}$.

To determine $\overline{\Delta s}$ and $\tilde{\Delta} F$ in terms of $\tilde{\Delta} \epsilon$, we solve equations (31) and (32) subject to the boundary conditions

$$
\frac{\tilde{\Delta} F}{F}=4 \frac{\tilde{\Delta s}}{c_{p}}+\tilde{\Delta} \eta,
$$

at $r=r_{t}$, and

$$
\bar{\Delta} s=0,
$$

at $r=r_{b}$. The upper boundary condition relates the tem-

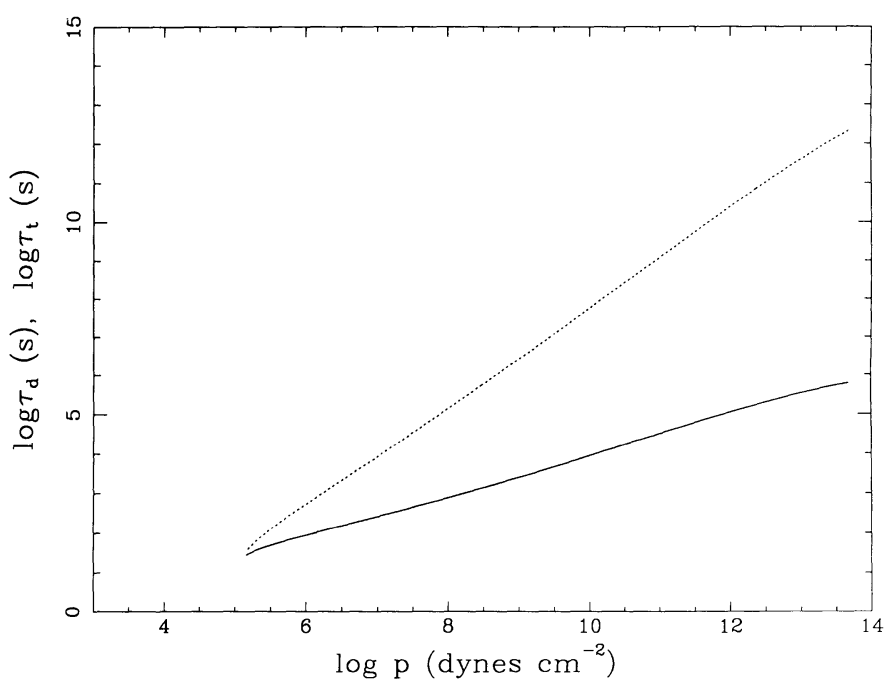

Fig. 8.- The diffusion time (solid curve) from equation (27) and the thermal relaxation time (dotted curve) from equation (28), plotted against the logarithm of the pressure.

perature perturbation at fixed $m, \tilde{\Delta} s / c_{p}$, to the perturbed flux, $\bar{\Delta} F$, radiated from the photosphere. It is a crude approximation that neglects perturbations of the radiative opacity and photospheric pressure. The parameter $\eta$ accounts for modification by the magnetic field of the radiative impedance between the top of the convection zone at $r_{t}$ and the photosphere at $R$. The excess flux associated with faculae is a prominent example of such an effect (Foukal \& Lean 1988). The lower boundary condition follows because $\tau_{t}\left(r_{b}\right)$ is much larger than $\tau_{c}$.

Integration of equation (31), with application of the lower boundary condition, yields

$$
\tilde{\Delta} s=\frac{2}{3} \int_{r_{b}}^{r} d r^{\prime}\left(\frac{\tilde{\Delta} F}{F}+\tilde{\Delta} \epsilon\right) \frac{\partial s}{\partial r^{\prime}} .
$$

We are interested in the entropy variations in the upper convection zone, which is where the perturbations implicated in the positive component of the frequency shifts arise. There, $\tau_{t}$ is much shorter than $\tau_{c}$, and as the energy equation (32) implies, $\widetilde{\Delta} F$ is independent of $r$. Since the magnitude of $\partial s / \partial r$ increases sharply with $r, \tilde{\Delta} F$ makes its contribution to the integral in equation (35) in the upper convection zone. We set $\widetilde{\Delta} F$ equal to its surface value and pull it outside the integral. ${ }^{10}$ When the upper boundary condition is applied to this simplified version of equation (35), the result is

$$
\frac{\tilde{\Delta} F}{F} \approx \frac{1}{1-8 \mathscr{D} s / 3 c_{p}}\left(\tilde{\Delta} \eta+\frac{8}{3 c_{p}} \int_{r_{b}}^{r_{t}} d r^{\prime} \tilde{\Delta} \epsilon \frac{\partial s}{\partial r^{\prime}}\right),
$$

where $\mathscr{D} s$ and $c_{p}$ are evaluated at $r_{t}$, and

$$
\frac{\tilde{\Delta} s}{c_{p}} \approx \frac{2 \mathscr{D} s}{3 c_{p}}\left(\frac{\tilde{\Delta} F}{F}+\frac{1}{\mathscr{D} s} \int_{r_{b}}^{r} d r^{\prime} \tilde{\Delta} \epsilon \frac{\partial s}{\partial r^{\prime}}\right),
$$

where $\mathscr{D} s$ and $c_{p}$ are evaluated at $r$.

Equation (36) reveals a serious problem. Using equation (26), we estimate

$$
\frac{\tilde{\Delta} F}{F} \sim-\left(\frac{\tilde{\Delta} B^{2}}{8 \pi \rho c^{2}}\right)\left(r_{t}\right)
$$

\footnotetext{
${ }^{10}$ Henceforth, $\triangle F$ denotes the perturbation of the emergent solar flux.
} 
for $\Delta \eta=0$. From the frequency shifts, we might have expected $\tilde{\Delta} F / F \sim-10^{-2}$. Instead, the ACRIM data suggest $\tilde{\Delta} F / F \lesssim$ $10^{-3}$

It is possible that magnetic fields decrease the radiative impedance of the photosphere so as to just slightly more than cancel the effects of the increased convective impedance. As this requires a cancellation of 1 part in 10, it seems unlikely. However, let us suppose that this remarkable cancellation really does take place. Then, using equation (37), and making the same approximations as in the derivation of equation (38), we obtain

$$
\frac{\tilde{\Delta s}}{c_{p}} \sim-\frac{\tilde{\Delta} B^{2}}{8 \pi \rho c^{2}}
$$

Substituting this expression into equation (14), we see that the entropy and magnetic perturbations produce frequency shifts of comparable size.

We cannot rule out the possibility that the flux perturbations parameterized by $\tilde{\Delta} \epsilon$ and $\tilde{\Delta} \eta$ cancel to high order. However, we would be surprised if they do. We think it more likely we have overestimated the degree to which magnetic stresses impede convection. Perhaps this is because we have not accounted for the ephemeral nature of the small-scale magnetic elements that dominate the mean square magnetic field. These block convective flux, but only for a short time. Most of the excess entropy that is stored during their brief lifetimes may work its way to the photosphere shortly after they disappear.

\section{DISCUSSION}

\subsection{Comparisons with Previous Work}

By now, the calculation of $p$-mode eigenfrequencies is a thriving industry. Most studies explore variants of spherical solar models. A sizable number treat the lifting of $m$ degeneracy by rotation. There are fewer investigations of models perturbed by internal magnetic fields, but these are becoming more fashionable. Those by Campbell \& Roberts (1989) and Evans \& Roberts (1990) are the closest in spirit to ours. Because both investigations predate the discovery of frequency shifts by Libbrecht \& Woodard (1990a, b), they lack the focus on the observational data that characterizes our work. Nevertheless, it is illuminating to review their results in light of ours.

Kuhn (1988) points out that latitude-dependent, solar cycle entropy variations might reconcile apparent disagreements among measurements of the even $m$ frequency splittings by different groups. His paper provides the first convincing indication that helioseismology is detecting changes associated with the solar cycle.

Campbell \& Roberts (1989) and Evans \& Roberts (1990) calculate eigenfrequencies for plane-parallel, solar-like models. The properties of the upper layers of their models are chosen to resemble those of the chromosphere. In Campbell \& Roberts (1989) the upper layer is threaded by a horizontal magnetic field whose strength declines with height in such a manner as to maintain a constant Alfvén speed. This magnetic field perturbation causes the mode frequencies to decrease. In Evans \& Roberts (1990) the magnetic field is again taken to be horizontal, but now of constant magnitude. Here it produces a positive frequency shift. How are we to understand these results?

We claim that a horizontal magnetic field must lower p-mode eigenfrequencies; the increase in path length is more important than the increase in propagation speed (see the dis- cussion at the end of $\S 2$ ). This is in accord with the finding by Campbell \& Roberts (1989). That Evans \& Roberts (1990) reach the opposite conclusion is understandable. A uniform magnetic field does not affect the equilibrium state of the atmosphere. It increases the propagation speed leaving the path length unchanged. Of course, a uniform magnetic field is not a realistic perturbation of a solar model since it requires an external stress to hold it in place.

Evans \& Roberts (1990) also investigate the effects of temperature changes on the mode frequencies. They find that the frequencies decline in response to an increase of the chromospheric temperature. This conclusion agrees with ours.

Gough \& Thompson (1988a) consider shallow, latitudedependent, magnetic perturbations as the cause of the symmetric (even $m$ ) component of the frequency splittings of the solar $p$-modes. They exclude thermal (entropy) perturbations because they would produce excessive latitude-dependent flux perturbations. In this respect their analysis is similar to that presented in $\S 4$.

Other papers consider frequency perturbations due to a toroidal magnetic field buried deep in the Sun, in particular, at or near the base of the convection zone where the solar dynamo is thought to operate (Vorontsov 1988; Gough \& Thompson 1988b, 1990; Dziembowski \& Goode 1988). Changes in the static structure of the Sun that must accompany magnetic variations are either not described or explicitly neglected. We find that frequency shifts due to changes in path length rival those due to changes in propagation speed. This issue aside, these papers make a couple of interesting points. There is general agreement that $10^{6} \mathrm{G}$ is the minimum detectable field at the base of the convection zone. Gough \& Thompson (1988b, 1990) and Vorontsov (1988) stress that localized fields produce frequency splittings that oscillate as a function of frequency. Such a concentrated field might account for the oscillatory component of the residuals in our fit to the frequency shifts shown in Figure $6 .^{11}$ The frequency spacing of the peak residuals would place the field at $p \approx 10^{8}$ dynes $\mathrm{cm}^{-2}$.

The chromospheric resonance is a well-established property of solar models (Ulrich \& Rhodes 1977; Ando \& Osaki 1977). Gouttebroze (1988) discusses helioseismic evidence for its reality. Perhaps the most convincing case for its existence is provided by the infrared heterodyne observations of an $\mathrm{OH}$ rotation line reported by Deming et al. (1988). They find a resonant frequency near $4.3 \mathrm{mHz}$.

\subsection{Some Loose Ends}

All of our results, and in particular equation (14), are based on perturbation theory. Since magnetic stresses in photospheric flux tubes are comparable to the local gas pressure, perturbation theory is not strictly applicable to them. Variations in the location of the transition layer also present problems for perturbation theory.

The shifts at the lowest frequencies demand that the mean square magnetic field strength perturbation increase with depth at least to the level where $p \approx 10^{8}$ dynes $\mathrm{cm}^{-2}$; $B^{2} \propto \rho^{1 / 3}$ works quite well. Shifts calculated for fields that do not increase with depth rise too rapidly with increasing frequency. This finding bears on the intermittency of the magnetic field in the convection zone. It suggests that the flux tubes

\footnotetext{
${ }^{11}$ Systematic errors in the data or their analysis seem a more plausible explanation.
} 
which pierce the photosphere continue to strengthen to greater depth than would be expected if they form by convective collapse.

Solar cycle variations of the eddy stress in the convection zone may be of comparable size to those of the magnetic stress. These changes will affect both propagation speed and path length. Unfortunately, it would be difficult, if not impossible, to determine even the sign of the resulting frequency shifts.

Adiabatic eigenfunctions are a poor approximation in a region starting just below the photosphere and continuing on out to the temperature minimum. Here, the radiative relaxation time is shorter than or comparable to the mode periods. Throughout much of this region it would be a better approximation to use the isothermal, rather than the adiabatic, sound speed in the wave equation. This correction would increase the calculated rate of growth with height of the mode amplitudes, especially at high frequencies. As a consequence, both the magnitude and height we deduce for the chromospheric temperature increase would be reduced.

The chromospheric temperature rise between 1986 and 1989 is poorly constrained owing to the uncertain frequency and strength of the chromospheric resonance. Because cooling by line emission provides a sensitive thermostat, the fractional increase in the heating rate is much larger than $\widetilde{\Delta} T / T$. No quantitative assessment of the variation of the chromospheric temperature seems to have been made, although chromospheric lines, including the $\mathrm{Ca}$ II $\mathrm{K}$ line, have been monitored over a magnetic cycle (White \& Livingston 1981). ${ }^{12}$ These data, obtained by viewing the Sun as a star, could be analyzed to determine the temperature variation as a function of solar cycle phase and chromospheric pressure.

\subsection{Conclusions}

We present a picture of the perturbations responsible for the $p$-mode frequency shifts. The positive shifts are due to an

\footnotetext{
12 A cursory examination of line profiles corresponding to different activity
} levels suggests that chromosphere heating varies by more than a factor of 2 . increase of the filling factor of small scale magnetic fields. A rise of the chromospheric temperature is responsible for the sharp cutoff to the positive shifts at high frequencies. The increase in mean square magnetic field strength and the temperature increase are shown in Figures 4 and 5. Our success in fitting the observed shifts with these perturbations is illustrated in Figure 7.

Perhaps our most important result is that the positive component of the $p$-mode frequency shifts can be accounted for by variations of the mean square magnetic field strength in the vicinity of the photosphere. A preliminary investigation, using full-disk magnetograms from Kitt Peak covering one day each in 1986 and 1988, appears to confirm our conclusions regarding mean square field changes derived from the frequency shifts. It is also notable that sunspots are a relatively minor contributor to the mean square magnetic field. Clearly, a more thorough comparison between the field changes deduced from the magnetograms and from the frequency shifts is warranted.

Our other conclusion, that increased magnetic activity is accompanied by higher temperature in the chromosphere, is not surprising. Unfortunately, the frequency shifts do not provide a precise measure of this rise, because they also depend on the uncertain properties of the chromospheric resonance.

The authors thank J. Harvey, K. Libbrecht, C. Thompson, $\mathrm{H}$. Wang, and M. Woodard for enlightening discussions. They are indebted to $\mathrm{J}$. Christensen-Dalsgaard for providing them with programs that generate his solar models and the equation of state on which they are based, and for helpful suggestions on improving the manuscript. This research was supported by NSF grant AST 89-13664 and NASA grant NAGW 1303. Part of it was performed while P. G. was visiting the HarvardSmithsonian Center for Astrophysics, and P. K. was visiting the Institute for Theoretical Physics at Santa Barbara. P. G. thanks the Smithsonian Institution of Washington for the award of a Regents Fellowship.

\section{APPENDIX}

Magnetohydrostatic equilibria satisfy

$$
\rho g+\nabla p=\frac{1}{c} \boldsymbol{j} \times \boldsymbol{B}
$$

where $4 \pi j=c \boldsymbol{\nabla} \times \boldsymbol{B}$ in the MHD limit.

Define a surface-averaged quantity as

$$
\langle Q\rangle(r) \equiv \int_{4 \pi} \frac{d \Omega}{4 \pi} Q(r) .
$$

Surface averaging enables us to express the Lorentz force in terms of the mean square field components. The averaged radial component of equation (A1), after an integration by parts with the constraint $\boldsymbol{\nabla} \cdot \boldsymbol{B} \equiv 0$, becomes

$$
\langle\rho\rangle g+\frac{\partial}{\partial r}\left(\langle p\rangle+\frac{\left\langle B_{h}^{2}\right\rangle}{8 \pi}-\frac{\left\langle B_{r}^{2}\right\rangle}{8 \pi}\right)=\frac{1}{4 \pi r}\left(2\left\langle B_{r}^{2}\right\rangle-\left\langle B_{h}^{2}\right\rangle\right)
$$

where $B_{h}$ and $B_{r}$ are the horizontal and radial components of the field, respectively.

The surface terms on the right hand side of equation (A3) are geometric in origin and may be neglected with respect to the gradient terms for fields whose scale length is much smaller than $R$. In this approximation, integration of equation (A3) subject to 
the boundary conditions, $\langle p\rangle(R)=\left\langle B_{r}^{2}\right\rangle(R)=\left\langle B_{h}^{2}\right\rangle(R)=0$, yields

$$
\langle p\rangle(r)+\frac{\left\langle B_{h}^{2}\right\rangle}{8 \pi}(r)-\frac{\left\langle B_{r}^{2}\right\rangle}{8 \pi}(r) \approx \frac{G M(M-m)}{4 \pi R^{4}},
$$

since near the surface,

$$
\int_{r}^{R} d r^{\prime}\langle p\rangle\left(r^{\prime}\right) \approx \frac{(M-m)}{4 \pi R^{2}}
$$

The magnetic terms in equation (A4) simplify on defining

$$
\left\langle B_{h}^{2}\right\rangle-\left\langle B_{r}^{2}\right\rangle \equiv \beta\left(\left\langle B_{r}^{2}\right\rangle+\left\langle B_{h}^{2}\right\rangle\right) .
$$

Note that $\beta$ takes on values between -1 and 1 . It is -1 for a pure radial field, 0 for a force-free field, $\frac{1}{3}$ for a statistically random field, and 1 for a pure horizontal field.

Ando, H., \& Osaki, Y. 1977, Pub. Astr. Soc. Japan, 29, 221

Campbell, W. R., \& Roberts, B. 1989, ApJ, 338, 538

Christensen-Dalsgaard, J. 1982, MNRAS, 199, 735

Cowling, T. G. 1941, MNRAS, 101, 367

Deming, D., Glenar, D. A., Kaufl, H. U., \& Espenak, F. 1988, in IAU Symposium 123, Advances in Helio- and Asteroseismology, ed. J. ChristensenDalsgaard \& S. Frandsen (Dordrecht: Reidel), p. 425

Dewar, R. L. 1970, Phys. Fluids, 13, 2710

Dziembowski, W., \& Goode, P. R. 1988, in IAU Symposium 123, Advances in Helio- and Asteroseismology, ed. J. Christensen-Dalsgaard \& S. Frandsen (Dordrecht: Reidel), p. 171

Evans, D. J., \& Roberts, B. 1990, ApJ, 356, 704

Foukal, P., \& Lean, J. 1988, ApJ, 328, 347

Gough, D. O., \& Thompson, M. J. 1988a, IAU Symposium 123, Advances in Helio- and Asteroseismology, ed. J. Christensen-Dalsgaard \& S. Frandsen (Dordrecht: Reidel), p. 175

. 1988b, IAU Symposium 123, Advances in Helio- and Asteroseismology, ed. J. Christensen-Dalsgaard \& S. Frandsen (Dordrecht: Reidel), p. 155

. 1990, MNRAS, 242, 25

\section{REFERENCES}

Gouttebroze, P. 1988, IAU Symposium 123, Advances in Helio- and Asteroseismology, ed. J. Christensen-Dalsgaard \& S. Frandsen (Dordrecht: Reidel), p. 421

Kuhn, J. R. 1988, ApJ, 331, L131

Ledoux, P., \& Walraven, T. 1958, Handbuch der Physik, vol 51, (Berlin: Springer), p. 353

Libbrecht, K. \& Woodard, M. 1990a, Nature, 345, 779

1990b, Proc. Santa Barbara Conf. on Intermediate Mass Stars

Newcomb, W. A. 1962, Nucl. Fusion Suppl., 2, 451

Spruit, H. C. 1990, in The Sun in Time, ed. C. Sonett (Tucson: University of Arizona Press), in press

Stenflo, J. O. 1989, Astr. Ap. Rev., 1, 3

Ulrich, R. K., \& Rhodes, E. J. 1977, ApJ, 218, 521

Vernazza, J. E., Avrett, E. H., \& Loeser, R. 1981, ApJS, 45, 635

Vorontsov, S. V. 1988, IAU Symposium No. 123, Advances in Helio- and Asteroseismology ed. J. Christensen-Dalsgaard \& S. Frandsen (Dordrecht: Reidel), p. 151

White, O. R., \& Livingston, W. C. 1981, ApJ, 249, 798

Willson, R. C., \& Hudson, H. S. 1988, Nature, 332, 810 